\title{
Ce-doped LuAG single-crystal fibers grown from the melt for high-energy physics
}

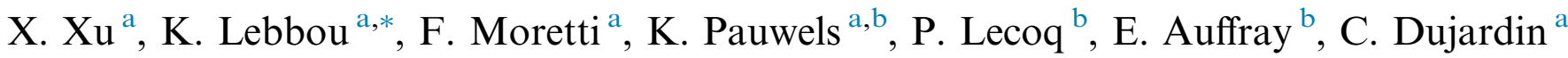 \\ ${ }^{a}$ Institut Lumière Matière, UMR5306 Université Lyon 1-CNRS, Villeurbanne Cedex, France \\ ${ }^{\mathrm{b}}$ European Organization for Nuclear Research, CERN, Dept PH-CHX-1211 Geneva, Switzerland
}

Received 12 September 2013; received in revised form 26 November 2013; accepted 28 December 2013

\begin{abstract}
Under a stationary stable regime undoped and Ce-doped $\mathrm{LuAG}\left(\mathrm{Lu}_{3} \mathrm{Al}_{5} \mathrm{O}_{12}\right)$ single-crystal fibers were grown by a micro-pullingdown technique. The meniscus length corresponding to the equilibrium state was $<200 \mu \mathrm{m}$. Fluctuations in the fiber composition and pulling rate were found to have a significant effect on the properties of the fibers grown. A great improvement in the performance was found in samples containing low Ce concentrations $(\leqslant 0.1$ at. $\%)$ and produced using pulling rates $<0.5 \mathrm{~mm} \mathrm{~min}^{-1}$. Under such conditions a good lateral surface fiber quality was obtained and light propagation was significantly improved. Conversely, a high Ce concentration and a high pulling rate resulted in a strong degradation of the fiber surface quality causing defects to appear and a decrease in light output.
\end{abstract}

(C) 2014 Acta Materialia Inc. Published by Elsevier Ltd. All rights reserved.

Keywords: Crystal; Fiber; Dopant; Attenuation

\section{Introduction}

Over the past few years, various scintillator materials have attracted much interest due to their importance in nuclear and particle physics research, and for medical and high-tech industrial applications [1]. Among various inorganic crystalline compositions, $\mathrm{Ce}^{3+}$-doped crystals, such as Ce: $\mathrm{Y}_{3} \mathrm{Al}_{5} \mathrm{O}_{12}$ [2], Ce: $\mathrm{YAlO}_{3}$ [3], Ce: $\mathrm{LiCaAlF}_{6}$ [4] and $\mathrm{Ce}: \mathrm{Lu}_{3} \mathrm{Al}_{5} \mathrm{O}_{12}$ [5-7], have been widely studied for their fast scintillation and high light yield. As one of the most popular crystals, Ce-doped $\mathrm{LuAG}\left(\mathrm{Lu}_{3} \mathrm{Al}_{5} \mathrm{O}_{12}\right)$ possesses certain some advantages, such as a high density of $6.7 \mathrm{~g} \mathrm{~cm}^{-3}$, high hardness, high effective $\mathrm{Z}$ number (62.9), short decay time ( $\sim 60 \mathrm{~ns})$, high light yield (26,000 photons $\mathrm{MeV}^{-1}$ ), and good mechanical and chemical stability [5-7]. LuAG has a garnet cubic-type structure $\left\{\mathrm{A}_{3}^{\mathrm{III}}\right\}\left[\mathrm{B}_{2}^{\mathrm{III}}\right]$ $\left(\mathrm{C}_{3}^{\mathrm{III}}\right) \mathrm{O}_{12}$ with space group Ia3d. This material belongs to the phase diagram of the $\mathrm{Lu}_{2} \mathrm{O}_{3}-\mathrm{Al}_{2} \mathrm{O}_{3}$ system [8]. The

\footnotetext{
* Corresponding author.

E-mail address: Kheirreddine.lebbou@univ-lyon1.fr (K. Lebbou).
}

garnet phase, $\mathrm{Lu}_{3} \mathrm{Al}_{5} \mathrm{O}_{12}$, is the most stable phase in this binary system. It can be easily obtained as a single phase via solid-state reaction. Its cubic eight-coordination is favorable for obtaining visible light emission [9]. The emission at $530 \mathrm{~nm}$ due to the $5 d^{1}-4 f$ radiative transition of $\mathrm{Ce}^{3+}$ matches well with the spectral sensitivity of photodetectors used in high-energy physics [10]. LuAG was reported as congruent material, so it is possible to grow undoped and $\mathrm{RE}^{3+}$-doped LuAG single crystals from the melt using Czochralski $(\mathrm{Cz})[11,12]$, Bridgman (Bd) $[13,14]$ and micro-pulling-down ( $\mu$-PD) $[15,16]$ techniques. Although the $\mathrm{Cz}$ method is the most convenient and well-developed method, it also has some drawbacks such as lower utilization ratio, serious segregation of dopants and high cost. Compared to the $\mathrm{Cz}$ method, the $\mu$-PD technique offers a way to cut production costs of materials when particular shapes (e.g. fibers, plates) are required. The high growth speed and high utilization ratio of $\mu-P D$ make this technique inherently cheaper in terms of building and running costs [17]. The samples can also be directly produced in a shape suitable for construction of detectors 
consisting of arrays of different crystals providing high detection granularity $[3,18]$. In this paper we report details of LuAG single-crystal fibers grown by the $\mu$-PD method, the performance of these fibers, and various optical and scintillation properties as a function of Ce concentration. The target is the development of a new generation of detectors for high-energy physics based on single-crystal fiber design $[18,19]$. The case for using inorganic scintillating fibers naturally arises since high detection granularity needs to be combined with high stopping power which can be obtained only with dense materials (the density of organic fibers being too small). In addition, the same scintillating fiber shape is of great interest for medical imaging (positron emission tomography) in a transverse configuration which requires small pixels combined with depth of interaction as well as good stopping power [20]. This area of interest is a spin-off activity from high-energy detection research. Our team is strongly involved in fiber crystal growth and through various fiber projects significant progress has been made to control the technology and to improve fiber quality and reproducibility in terms of optical performance.

\section{Experimental}

Undoped and Ce-doped LuAG $\left(\mathrm{Lu}_{3(1-x)} \mathrm{Ce}_{3 x} \mathrm{Al}_{5} \mathrm{O}_{12}\right)$ single-crystal fibers were grown by the $\mu$-PD technique. Details of the $\mu$-PD technique were described in detail in Refs. [21-23]. The initial raw materials were undoped and Ce-doped crackle LuAG crystals grown by the $\mathrm{Cz}$ method, and single-phase LuAG prepared by solid-state reaction at $1400{ }^{\circ} \mathrm{C}$. The charge is contained in an iridium crucible die of outer diameter $2 \mathrm{~mm}$. The melt flows through a hole in the center of the die $\sim 0.6 \mathrm{~mm}$ in diameter. Undoped $\langle 111\rangle$ oriented seed was used at the bottom of the capillary die and pulled down continuously with a pulling rate ranging from 0.2 to $0.75 \mathrm{~mm} \mathrm{~min}^{-1}$. The molten zone, the meniscus and part of the grown crystal were directly observed and monitored with a CCD camera. The morphology and surface quality of the fibers were longitudinally and transversally analyzed using a Leica optical microscope. An experiment to determine the attenuation of luminescence was carried out along grown fibers of LuAG. The luminescence was obtained using a Xe lamp as excitation source. The Xe lamp source beam dimensions were determined by two slits and the spot on the fiber was set at $1 \times 1 \mathrm{~mm}^{2}$. The fiber absorbed the photons and scintillation light propagated along the fiber. The photoluminescence was collected by a bundle of optical fibers set on one side of the crystal. The signal from the optical fiber was then spectrally analyzed by a monochromator (Triax series 320) and a CCD 3000 camera. The fiber was then moved perpendicularly to the Xe lamp photon and luminescence spectra were recorded.

\section{Results and discussion}

\subsection{Crystal growth}

Several crucible capillary die designs have been tested to find the best growth conditions to produce fibers with stable diameters. Because of the high wettability of the LuAG melt, the standard conical crucible (Fig. 1a) did not allow fibers of stable diameter to be pulled. Using this crucible shape, the melt flow through the capillary die periphery and the diameter increased continuously (Fig. 1b) leading to large crystals containing cracks. On the other hand, using a crucible with its capillary die perpendicular to the conical part of the crucible (Fig. 1c) proved successful at stabilizing the growth process. Initially
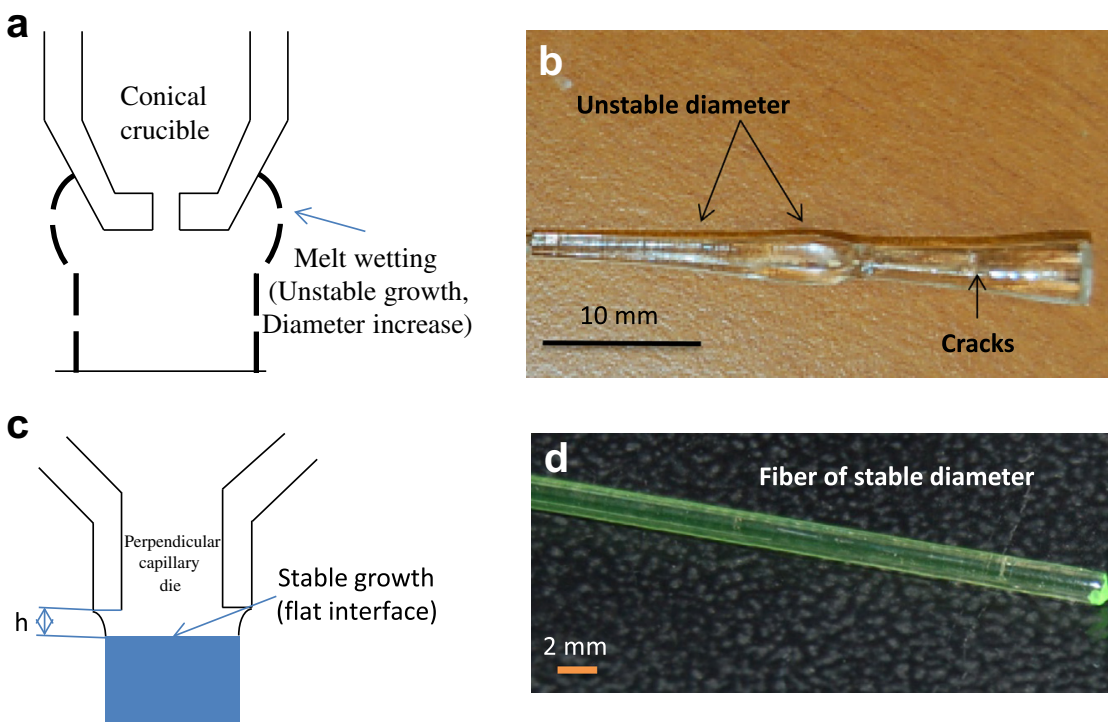

Fig. 1. Crucible design for growing undoped Ce-doped LuAG fiber under a stationary stable regime and the conditions for obtaining fibers of stable diameter. 
Table 1

LuAG fibers as a function of growth conditions and their appearance.

\begin{tabular}{lllll}
\hline Reference & $\begin{array}{l}\text { Starting raw } \\
\text { materials }\end{array}$ & $\begin{array}{l}\text { Doping Ce level } \\
(\text { at.\%) }\end{array}$ & $\begin{array}{l}\text { Pulling rate } \\
\left.(\mathrm{mm} \mathrm{min})^{-1}\right)\end{array}$ & Appearance \\
\hline LuAG B1 & CZ Crackle & 0 & 0.5 & Transparent, colorless \\
LuAG C1 & Cz Crackle & $<0.05$ & 0.25 & $\begin{array}{l}\text { Yellow, transparent, bubble free, good surface quality, good light } \\
\text { propagation }\end{array}$ \\
LuAG C2 & Cz Crackle & $<0.05$ & 0.5 & Yellow, transparent, contains bubbles \\
LuAG C3 & Cz Crackle & $<0.05$ & 0.75 & Yellow, contains bubbles, low light propagation \\
LuAG C4 & Oxydes & 0.02 & 0.32 & Yellow, transparent, good surface quality, good light propagation \\
LuAG C5 & Oxydes & 0.05 & 0.5 & Yellow \\
LuAG C8 & Oxydes & 0.1 & 0.6 & Slightly green, transparent, contains bubbles \\
LuAG C13 & Cz Crackle & 0.1 & 0.6 & Slightly green, transparent, contains bubbles \\
LuAG C15 & Cz Crackle & 0.1 & 0.65 & Slightly green, transparent, contains bubbles \\
LuAG C18 & Cz Crackle & 0.1 & 0.5 & Slightly green, transparent, contains bubbles \\
LuAG C20 & Cz Crackle & 0.1 & 0.25 & Yellow, transparent, good surface quality, good light propagation \\
LuAG C21 & Cz Crackle & 0.1 & 0.75 & Slightly green, transparent, contains bubbles \\
\hline
\end{tabular}

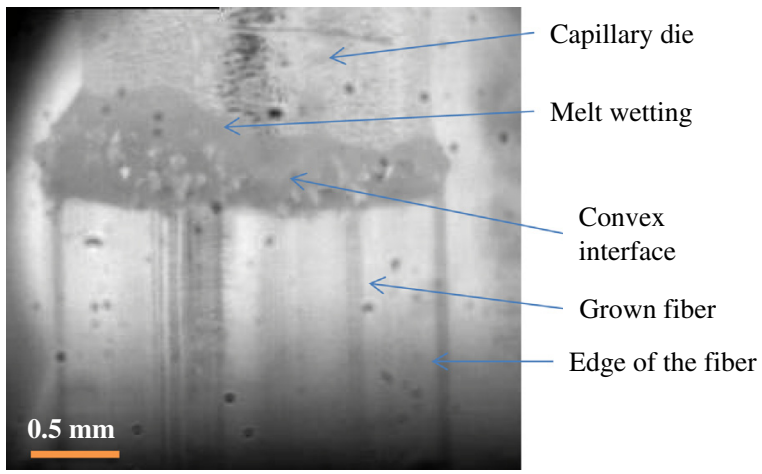

Fig. 2. Ce (0.12 at.\%) LuAG fiber growth showing convex crystallization interface and strong wetting on the periphery of the capillary die.

a drop at the capillary die covers the lip of the circular capillary channel and a fiber single crystal grows. A $\langle 111\rangle$ LuAG seed crystal $200 \mathrm{~mm}$ long is dipped in the melt at the bottom of the crucible in the capillary die and the melt temperature is adjusted until a meniscus is supported. The growth process was controlled manually by changing the pulling rate and the heating power, using as a guide the ratio of meniscus height $(h)$, defined as the height of the bright region under the crucible, to fiber diameter (Fig. 1c). Experimentally, in the case of LuAG melt, in a stationary state, this ratio was found to be 1:5-10 (meniscus height/fiber diameter) depending on the growth parameters, crucible design (shape factor) and starting composition. Despite these fibers being grown in iridium crucibles, they do not contain Ir ions.

The crystal fibers were grown at a pulling-down rate in the range $0.2-0.75 \mathrm{~mm} \mathrm{~min}^{-1}$ and were $2.2 \mathrm{~mm}$ in diameter, close to the diameter of the crucible opening (Fig. 1d). Table 1 summarizes the results as a function of the growth conditions. The typical length of the molten zone corresponding to the stationary state was $\sim 170 \mu \mathrm{m}$ and the crystallization interface was flat and stable. The length of the crystals was up to $400 \mathrm{~mm}$, and $100 \%$ of the liquid was crystallized into fibers. Irrespective of the starting composition used in this work $\left(\mathrm{Ce}^{3+} \leqslant 0.1\right.$ at.\%), we did not observe any composition evolution resulting in the formation of a second phase. Disconnection of the fiber growing from the molten zone was never observed even when changing the growth parameters such as the pulling rate. At high $\mathrm{Ce}$ concentrations $(\mathrm{Ce}>0.1$ at.\%) there is an increase in liquid wetting, the interface becomes convex (Fig. 2) and the diameter increases up to $2.5 \mathrm{~mm}$. Fig. 3a shows undoped LuAG fibers and Fig. 3b shows 0.06 at.\% Ce-doped LuAG fibers. The undoped fibers are transparent, colorless and display a smooth surface. The Ce-doped fibers are transparent and show a color variation from colorless to green depending on the starting Ce concentration in the melt (Fig. 4). This is due to a strong intensity increase and a broadening of $\mathrm{Ce}^{3+}$-associated absorption bands observed with increasing dopant concentration

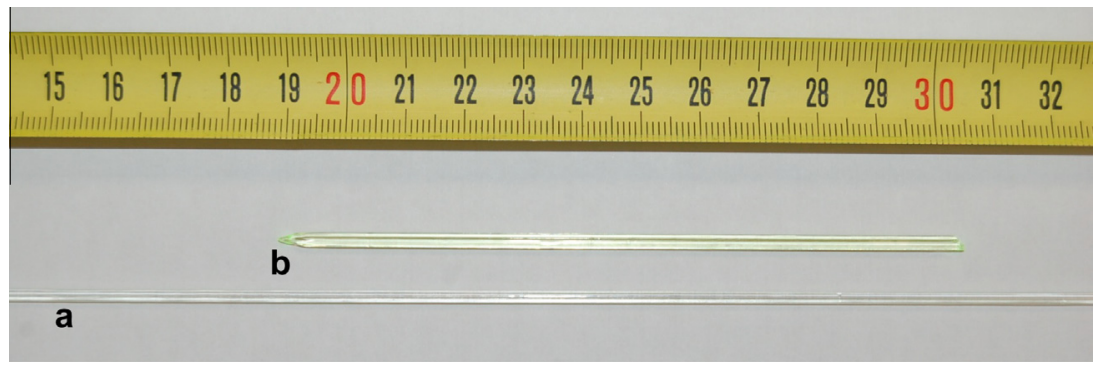

Fig. 3. (a) Undoped LuAG and 0.06 at.\% Ce-doped LuAG grown under a stationary stable regime (pulling rate $0.3 \mathrm{~mm} \mathrm{~min}^{-1}$ ). 


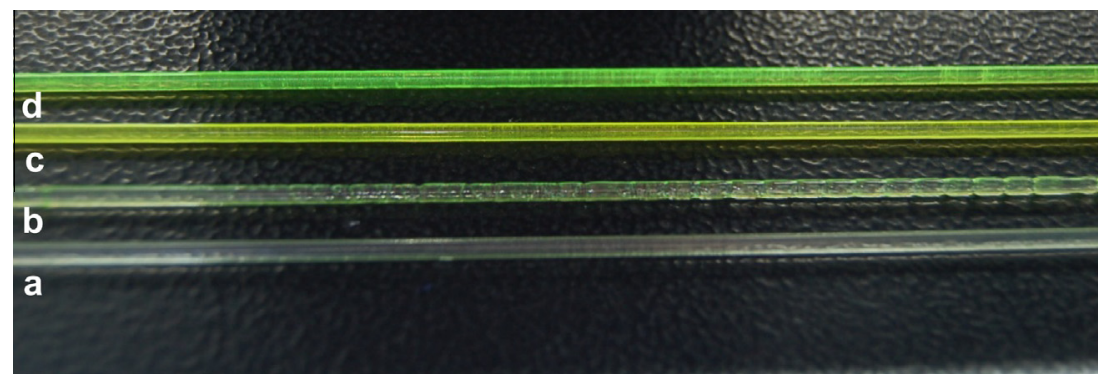

Fig. 4. Color contrast evolution as a function of Ce concentration: (a) undoped LuAG; (b) Ce $=0.03$ at. $\%$; (c) Ce $=0.06$ at. $\%$. The green symbols in (a) indicate high Ce concentration $(0.12$ at.\%). Note the starting Ce concentration in the melt. (For interpretation of the references to color in this figure legend, the reader is referred to the web version of this article.)

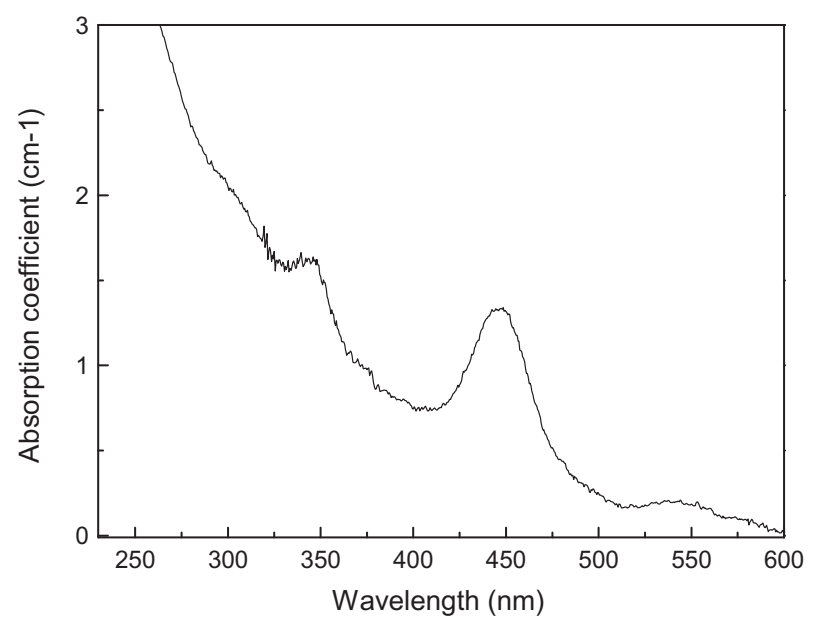

Fig. 5. Absorption spectra of 0.06 at.\% Ce-doped LuAG performed at room temperature.

(Fig. 5). Different Ce-LuAG pieces were transversally cut from the grown fibers and analyzed by optical microscopy (Fig. 6). All the grown fibers with high $\mathrm{Ce}(>0.1$ at.\%) concentration show strong Ce radial segregation. This is principally due to difference of the $\mathrm{Ce}^{3+}$ size $(103.4 \mathrm{pm})$ which is considerably greater than the size of $\mathrm{Lu}^{3+}$ $(86.1 \mathrm{pm})$ [24]. Fig. $7 \mathrm{a}$ and $\mathrm{b}$ shows a transverse

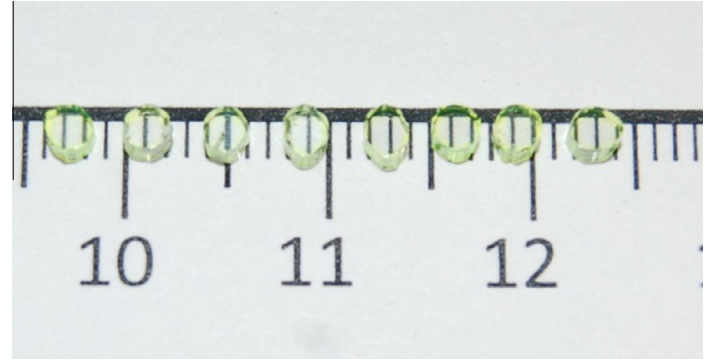

Fig. 6. Cross-section of the Ce-doped LuAG optically polished for microscopy analysis. The fibers were transparent and do not contain visible defects.

cross-section of a Ce-doped LuAG fiber grown from starting melt containing 0.12 at.\% Ce. The color contrast is sensitive to the chemical composition. A visible concentration gradient can be seen in this sample. The fiber periphery was brighter than the center, which confirms the Ce segregation in the periphery of the fiber. The longitudinal Ce composition analysis along the fiber axis shows a constant concentration. This is related to the diffusive transport regime in the capillary die. Because of the relatively high pulling speed and no mixing in the capillary channel (no convection), the diffusion length $l=D / v(v$, growth rate; $D$, diffusion speed) is smaller than the inner length of the crucible capillary $(2.5 \mathrm{~mm})$. Therefore we did not observe any

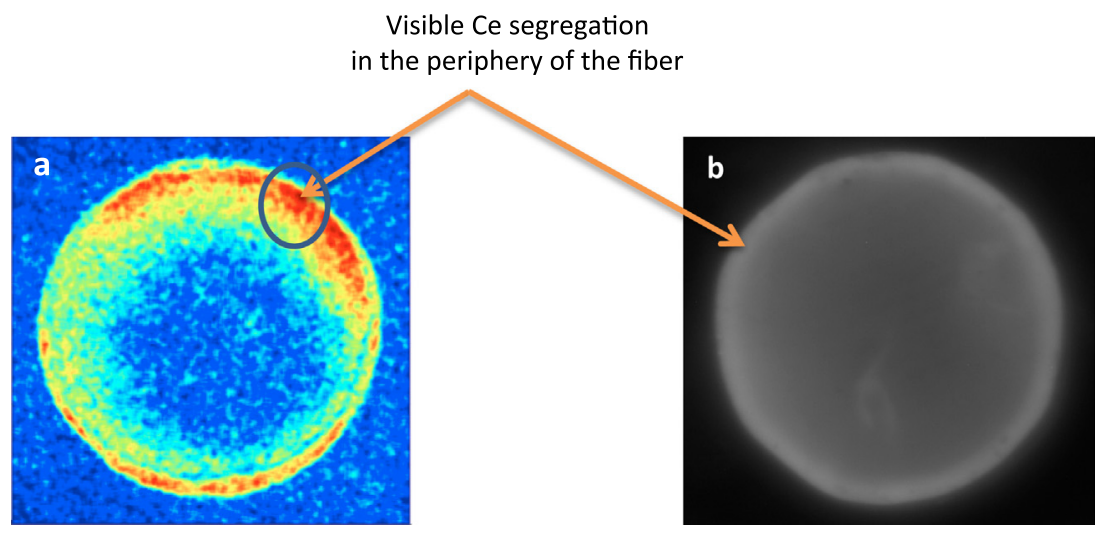

Fig. 7. Cross section image of the 0.12 at.\% Ce-doped LuAG fibers excited under X-rays (a) and electrons (b), showing a gradient concentration of Ce dopant clearly visible in the periphery. 

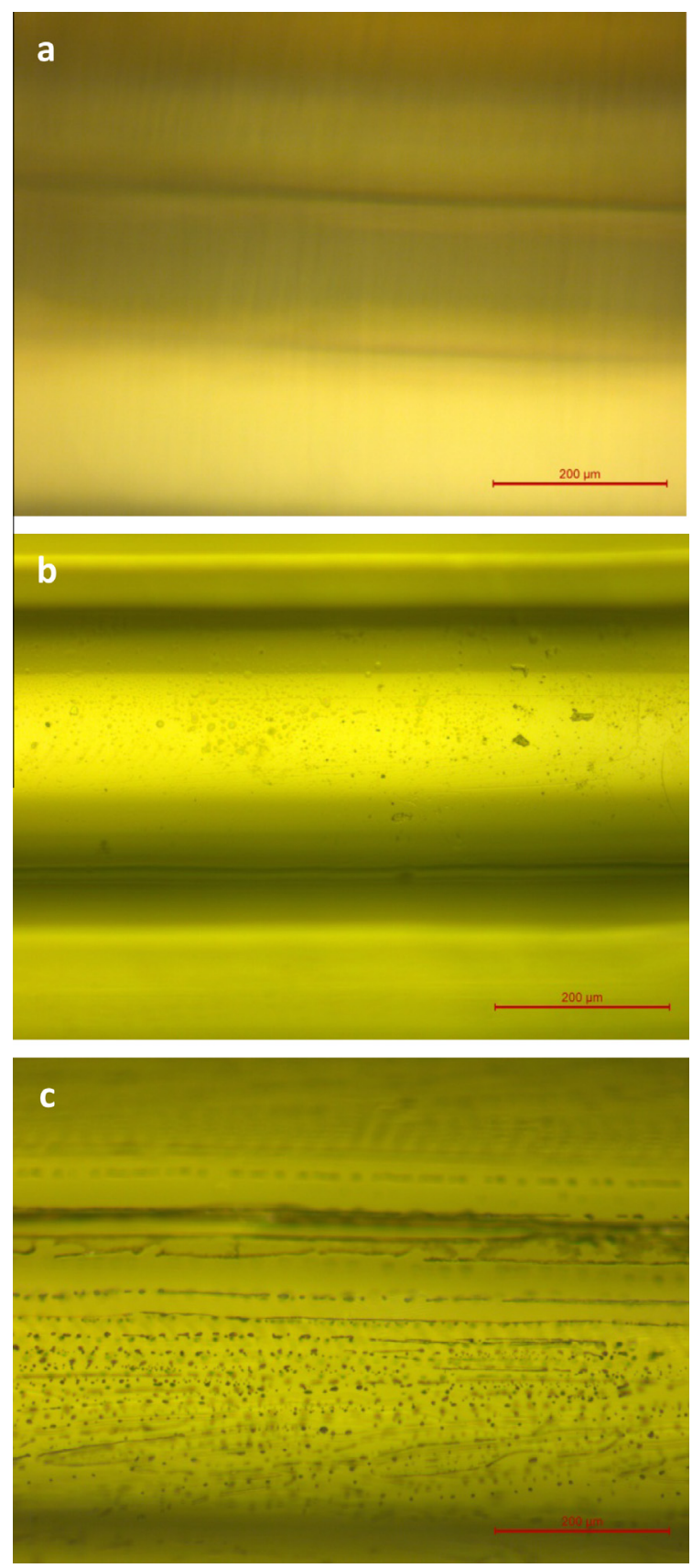

Fig. 8. Analysis of the bubbles at the lateral periphery of the fibers grown: (a) undoped LuAG; (b) $\mathrm{Ce}=0.06$ at. $\%$; and (c) $\mathrm{Ce}=0.12$ at. $\%$.

fluctuation in Ce composition along the fiber axis. In the case of garnet materials, it is common to observe defects in crystals grown from a melt due to gas bubbles which appear at some critical supersaturation of the melt resulting from a gas impurity whose distribution coefficient is usually $<1$. Depending on the starting $\mathrm{Ce}$ concentration in the melt, gas bubbles become trapped in the liquid. The segregation of these bubbles occurs during the liquid-solid transition, leading to an increase in the bubble size and their subsequent movement upwards, driven by buoyancy. In garnet materials, the dependence of gas bubbles on the dopant distribution is not clear. For

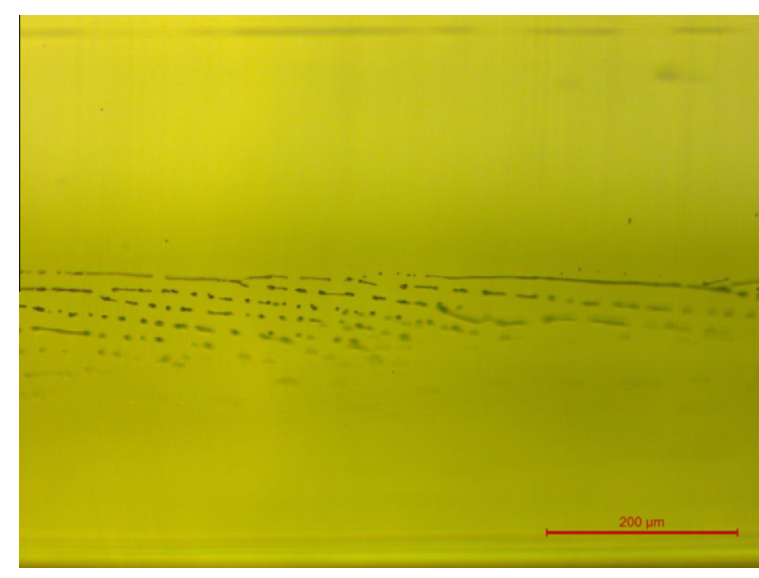

Fig. 9. Bubbles well aligned along the growth direction.
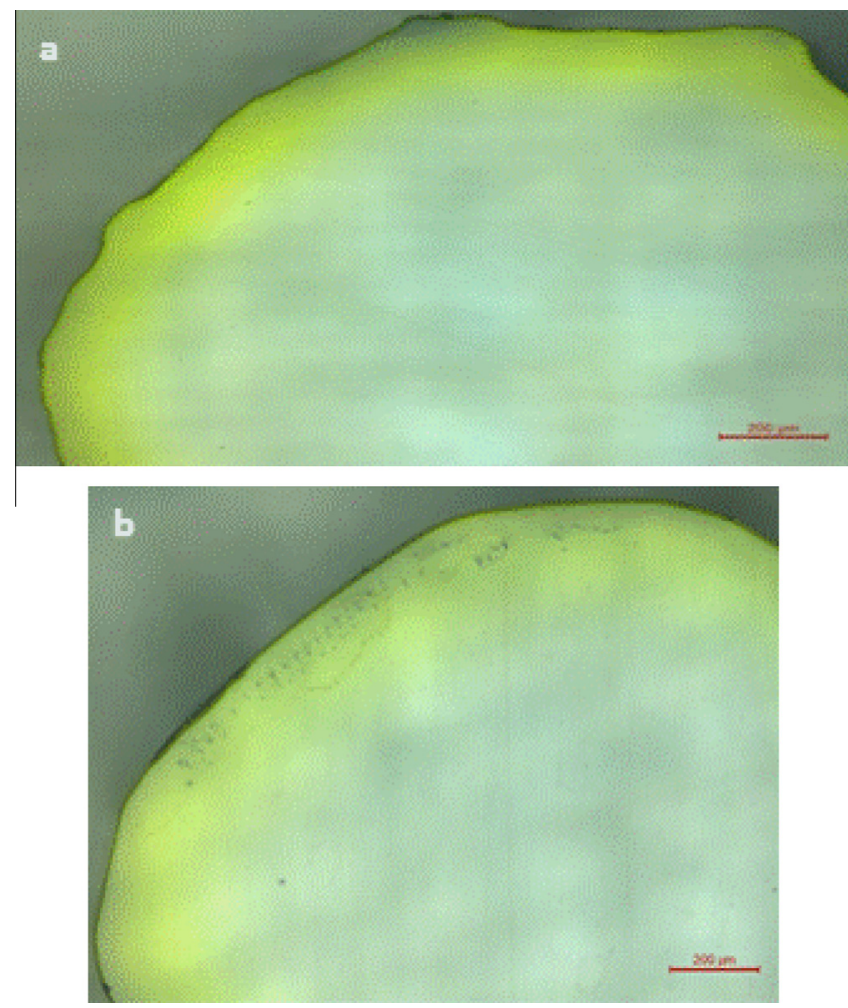

Fig. 10. Bubbles distribution in the cross-section of the Ce-doped LuAG: (a) undoped LuAG, pulling rate $=0.3 \mathrm{~mm} \mathrm{~min}^{-1}$; (b) $\mathrm{Ce}=0.06$ at. $\%$, pulling rate $=0.3 \mathrm{~mm} \mathrm{~min}^{-1}$.

undoped LuAG crystals, regardless of the pulling rate $\left(V \leqslant 0.3 \mathrm{~mm} \mathrm{~min}^{-1}\right)$ no bubbles are observed (Fig. 8a). On the other hand, highly Ce-doped LuAG fibers exhibit bubbles along the growth direction (Fig. $8 \mathrm{~b}$ and c). As we have already shown in our previous paper [22], most bubbles have a spherical geometry and some are elongated along the growth direction. An analogous situation has been observed when growing sapphire fibers using the same capillary channel $[22,23]$. Optical microscopy shows that the bubbles were well aligned along the fiber axis (Fig. 9). 

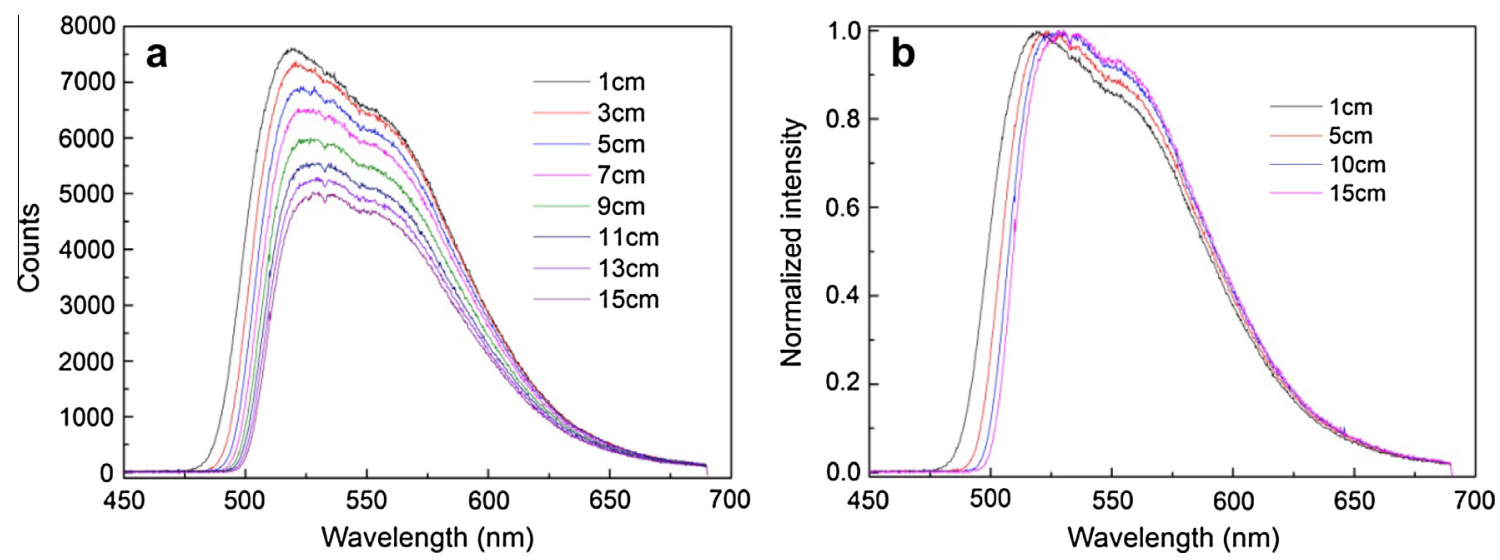

Fig. 11. (a) Photoluminescence spectra as function of the interacting position for fiber LuAG C1. (b) Comparison of the emission spectra shape (after normalization) for $1,5,15$ and $20 \mathrm{~cm}$ position from the output side.

For a low pulling rate $\left(0.3<V<0.5 \mathrm{~mm} \mathrm{~min}^{-1}\right)$ and low Ce concentration in the melt $(<0.1$ at. $\%)$, the crystallization interface is planar and there is only one lateral layer of bubbles. The average maximum distance from the periphery to the bubbles is $\sim 93 \mu \mathrm{m}$ (Fig. 10). However, the rest of the crystal volume is bubble free. The behavior is similar for the different parts of the fibers (beginning, middle and the end). These conditions ensure a low level of bubbles, which are only formed in the regions with a minimum component of the melt velocity ahead of the solidification interface. At velocities higher than the critical maximum growth rate, constitutional undercooling generates bubble propagation. The fibers grown with a low pulling rate have a constant diameter and a smooth surface. This is mainly related to the stable molten zone and the interface. Hence, it is very important to determine the optimum regime in which fibers can be pulled without bubbles forming. The main problem in producing high-quality Ce-doped LuAG is how to prevent the formation of bubbles, which form in the region where the melt velocity ahead the crystallization interface is minimum.

\subsection{Light propagation}

The optical attenuation of scintillation light within a fiber is a critical parameter, since the scintillating signal has to propagate along the entire length of the fiber. The optical attenuation is related to the crystal quality and surface roughness. Defects play an important role in the quality of the propagation of scintillation light. In order to estimate the attenuation length of the fibers, we excited them at different locations with a UV beam.

When photons irradiate a fiber, scintillation light propagates in two opposite directions. Therefore the propagation properties become crucial in order to detect as much light as possible at each end of the fiber. We also measured the attenuation property of a standard commercial plastic scintillator fiber for comparison. For each interacting position, the photoluminescence spectrum is recorded and the set of measurements is shown in
Fig. 11. A change in the shape of the photoluminescence spectra as a function of the excitation position can be seen. When the interaction position was moved away from one end of the fiber, the intensity of luminescence decreased and the emission bands shifted to longer wavelengths. As reported before, there are two kinds of propagation losses $[6,12]$. One is self-absorption due to the overlap between emission and absorption. The self-absorption phenomenon acts principally on the shortest wavelength part of the emission spectra. The shape of the emission spectra is then changed. Another propagation loss is due to reflection losses and uniformity along the fiber. The nearest position from the collection light side is considered as the initial emission $\left(I_{0}\right)$. The function $I(x)$ is presented on a log scale in Fig. 12. It shows the attenuation curves of the Ce-doped LuAG fibers as a function of the pulling rate. The measurements were performed under visible light $(450 \mathrm{~nm})$. With such photons it is only possible to probe the propagation of photons emitted by the Ce sites. The light propagation in LuAG $\mathrm{C} 1\left(v=0.25 \mathrm{~mm} \mathrm{~min}^{-1}\right.$, Fig. 12) is much better than those of the calibrated plastic fiber and the fibers grown with higher pulling rates. We note also the good

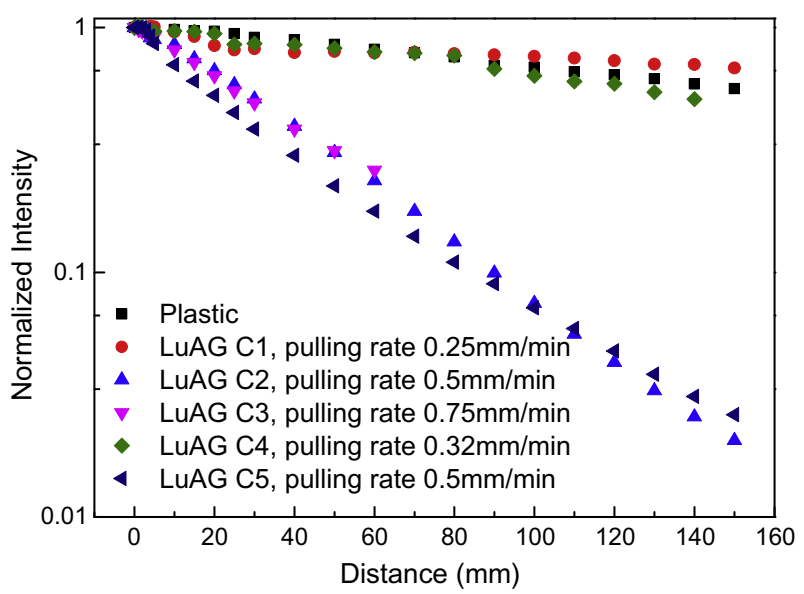

Fig. 12. Attenuation curves for plastics fiber and Ce $(<1$ at.\%)-doped LuAG fibers as a function of pulling rate. 
light propagation in LuAG $\mathrm{C} 4 \quad\left(v=0.32 \mathrm{~mm} \mathrm{~min}^{-1}\right.$, Fig. 12) similar to the calibrated plastic fiber. On the other hand, the light is considerably attenuated for fibers grown with pulling rates $>0.32 \mathrm{~mm} \mathrm{~min}^{-1}$. Optical microscopy characterization showed that the surface quality of LuAG $\mathrm{C} 1$ and LuAG C4 is better than that of fibers grown with higher pulling rates $\left(v>0.32 \mathrm{~mm} \mathrm{~min}^{-1}\right)$; in particular, the presence of bubbles explains the attenuation results obtained. The comparison of light attenuation allows us to conclude that a good surface quality is crucial for obtaining reasonable light attenuation lengths. Surface diffusion and bubbles are otherwise favoring escape of light through lateral faces. Therefore, growing Ce-doped LuAG fibers with low pulling rates is essential to produce fibers that exhibit good optical quality.

\section{Conclusion}

We have demonstrated that by the optimization of the pulling rate and Ce concentration of LuAG single-crystal fibers grown by $\mu-\mathrm{PD}$, a significant improvement in the optical quality of these fibers can be achieved. Low Ce concentration ( $\leqslant 0.1$ at. $\%)$ and low pulling rate $(\leqslant 0.32 \mathrm{~mm} \min$ ${ }^{-1}$ ) were identified as a good compromise to obtain good surface quality and light propagation. A high pulling rate $\left(>0.32 \mathrm{~mm} \mathrm{~min}^{-1}\right)$ and high Ce concentration $(>0.1$ at. $\%)$ strongly affect the surface quality and increase light attenuation through the fiber. Because of the progress made in the optimization of Ce-doped LuAG starting raw materials and the growth parameters, the next step will be focused on possible designs of practical calorimeters based on fiber design, and will address the different challenges related to high-energy physics.

\section{Acknowledgments}

This work was supported by the French National Agency for Research under grant agreement ANR-10BLAN-0947 (INFINHI), and the European Union FP7/ 2007-2013 under grant agreements 256984-EndoTOF-
PET-US and 295025-IPERA. The authors are grateful to Cristal innov for fiber polishing.

\section{References}

[1] Nikl M. Meas Sci Technol 2006;17:R37.

[2] Moszynski M, Ludziejewski T, Wolski D, Klamra W, Norlin LO. Nucl Instrum Methods A 1994;345:461.

[3] Alshourbagy M, Bigotta S, Herbert D, Del Guerra A, Toncelli A, Tonelli M. J Cryst Growth 2007;303:500.

[4] Yamaji A, Yokota Y, Yanagida T, Kawaguchi N, Futami Y, Fujimoto Y, et al. J Cryst Growth 2012;352:1069.

[5] Derdzyan MV, Ovanesyan KL, Petrosyan AG, Belsky A, Dujardin C, Pedrini C, et al. J Cryst Growth 2012;361:212.

[6] Dujardin C, Mancini C, Amans D, Ledoux G, Abler D, Auffray E, et al. J Appl Phys 2010;108:013510.

[7] Petrosyan AG, Ovanesyan KL, Sargsyan RV, Shirinyan GO, Abler D, Auffray E, et al. J Cryst Growth 2010;312:3136.

[8] Shirvinskaya AK, Popova VF. Dokl Akad Nauk SSSR 1977;223:1110.

[9] Blasse G, Bril A. J Chem Phys 1967;47:5139.

[10] Di J, Xu X, Xia C, Li D, Zhou D, Wu F, et al. J Cryst Growth 2012;351:165-8.

[11] Kamada K, Yanagida T, Endo T, Tsutumi K, Yoshino M, Kataoka J, et al. J Cryst Growth 2012;352:91.

[12] Sugiyama M, Fujimoto Y, Yanagida T, Yamaji A, Yokota Y, Yoshikawa A. J Cryst Growth 2013;362:178.

[13] Petrosyan AG. J Cryst Growth 1994;139:372.

[14] Petrosyan AG, Popova VF, Gusarov VV, Shirinyan GO, Pedrini C, Lecoq P. J Cryst Growth 2006;393:74.

[15] Sangla D, Aubry N, Nehari A, Brenier A, Lebbou K, Balembois F, et al. J Cryst Growth 2009;312:125.

[16] Sugiyama M, Fujimoto Y, Yanagida T, Yokota Y, Pejchal J, Furuya Y, et al. J Cryst Growth 2011;33:905.

[17] Yoshikawa A, Nikl M, Boulon G, Fukuda T. Opt Mater 2007;30:6.

[18] Lecoq P. J Phys Conf Ser 2009;160:012016.

[19] Pauwels K, Dujardin C, Gundacker S, Lebbou K, Lecoq P, Lucchini M, et al. JINST 2013;8:P09019.

[20] Braem A. Nucl Instrum Methods Phys Res A 2004;A525:268.

[21] Lebbou K, Perrodin D, Fukuda T, Chani V, editors. Advances in materials research, shaped crystal growth by micro-pulling-down technique. Berlin: Springer-Verlag; 2007. p. 173-86.

[22] Ghezal EA, Li H, Nehari A, Alombert-Goget G, Brenier A, Lebbou K, et al. Cryst Growth Des 2012;12:4098.

[23] Ghezal EA, Nehari A, Lebbou K, Duffar T. Cryst Growth Des 2012;12(11):5715.

[24] Shannon RD, Prewit CT. Acta Crystallogr 1969;B25:925. 